\author{
Joachim Hötzel
}

\title{
Anthroposophisch erweiterte Kardiologie: Erfahrungen und Praxisbeispiele
}

Seit 10 Jahren verknüpfe ich die konventionelle Kardiologie mit der Anthroposophischen Medizin. In diesem Zeitraum habe ich mehrere tausend Patienten gesehen, die in der Regel gezielt eine erweiterte Form der Kardiologie suchen. Motivation und Fragestellungen dieser Patienten sind sehr vielfältig:

- Unverträglichkeit, Sorge vor Nebenwirkungen der konventionellen Therapie - gibt es anthroposophische Heilmittel?

- Verbundenheit mit der Naturheilkunde, speziell mit der Anthroposophischen Medizin.

- Grundlegend kritische Einstellung gegenüber dem konventionellen Medizinbetrieb, in dem der Patient als Persönlichkeit nicht wahrgenommen wird.

- Zweitmeinung z.B. zu Operationsindikationen ( Brauche ich die neue Klappe wirklich?»), nach mehreren frustranen Interventionen, Pulmonalvenenisolation bei Vorhofflimmern ( Was kann die Anthroposophische Medizin?»), zur prophylaktischen Indikation eines automatischen implantierbaren Kardioverter-Defibrillators (AICD), zur «LifeVest», zur Entfernung eines «Assist Device» bei Zustand nach Myokarditis usw.

Das Zeitbudget für eine Erstuntersuchung liegt bei ca. 2 h. Neben den konventionellen Aspekten beinhaltet die umfassende Anamnese regelhaft auch Fragen nach der Biografie, nach übergeordneten Rhythmen (Verdauung/Schlafrhythmus/regenerative Rhythmen), nach dem Wärmeorga- nismus und natürlich die von Rudolf Steiner angegebenen "Anamnesefragen» aus dem 1. Medizinerkurs [1].

Der Anamnese folgen körperliche und notwendige technische Untersuchungen (Echo/Ischämietest/Gefässdiagnostik usw.)

Änderungen im Lebensstil sind der zentrale therapeutische Ansatz; ca. 90\% der Patientin erhalten zusätzlich eine anthroposophische Therapie (Heilmittel/Heileurythmie) entweder ausschliesslich oder in Kombination mit einer konventionellen Medikation.

Jeder Patient bzw. der Hausarzt bekommt einen ausführlichen Arztbrief. Er wird aufgefordert, nach 4-6 Wochen Rückmeldung zu geben zum weiteren Verlauf unter der geänderten Therapie (per E-Mail oder Telefon). Genaue Zahlen zum Therapieerfolg gibt es nicht, doch der Zufriedenheitsgrad (Symptomverbesserung) ist ausserordentlich hoch; auch sehr komplexe Situationen (Rhythmusstörungen/Herzinsuffizienz/koronare Herzkrankheit (KHK)) lassen sich über Jahre stabil halten entweder ohne oder mit deutlich reduzierter konventioneller Therapie.

Die Praxis liegt weit abgeschieden an einem Wirtschaftsweg im ländlichen Raum. Das Einzugsgebiet ist überregional (bundesweit), was sicher auch der Tatsache geschuldet ist, dass es nur wenige anthroposophische Kardiologen gibt. Ich hatte das Privileg, die anthroposophische Kardiologie «hands-on» in der Klinik Arlesheim (Leitender Kardiologe: Dr. Christoph Kaufmann) zu erlernen. Inzwischen gibt es jedoch auch spezielle kardiolo- gisch-anthroposophische Literatur, die den Einstieg in das menschenkundliche Verständnis spezieller kardiologischer Krankheitsbilder und deren Therapie wesentlich erleichtert [2-6].

Eine Kardiologie ohne den erweiterten Blick auf den Patienten und ohne die erweiterten Therapiemöglichkeiten ist für mich persönlich nicht mehr vorstellbar.

Im Folgenden werden drei stark komprimierte Krankengeschichten aus dem Alltag einer kardiologischen Praxis geschildert:

\section{«Funktionelle» \\ Herzbeschwerden}

\section{D.S., 53 Jahre (w); Erstkonsultation 19.05.2015}

- Aktuelle Beschwerden: Seit ca. 2 Monaten bei stärkeren Belastungen, bei Aufregung Dyspnoe, auch eine Art von thorakaler Beklemmung, dies sei bedrohlich, kein Schmerz. Gelegentlich Schwindel, Palpitationen, Pulsfrequenzen um $100 / \mathrm{min}$ in Ruhe, insbesondere im Rahmen von Hitzewallungen (Menopause seit ca. 1 Jahr).

- Frage der Patientin bzw. des überweisenden Hausarztes: Abklärung, Therapie?

- Weitere Vorgeschichte: Generalisierte Arthrose, teils aktiviert, HWS betont. Früher auch die Diagnose Fibromyalgie.VieleMuskelschmerzen. 2011 Hysterektomie wegen schwerer Blutungen. Status nach zwei Knieoperationen links.

\section{KARGER}

(c) 2017 S. Karger GmbH, Freiburg 
- Systemanamnese: Vermehrt kalte Hände und Füsse, Schlaf insgesamt gut, wenig Traumleben, Präferenz für Süsses, Verdauung gut, Appetit okay, normales Durstempfinden.

- Kardiovaskuläres Risiko: Keins.

- Biografie: Geboren auf Sizilien, mit 6 Jahren Umsiedlung nach Deutschland, das zweite von vier Kindern. Grundschule, Ausbildung zur Friseurin. Heirat mit 19 Jahren, zusammen mit dem Ehemann Aufbau eines Eiscafés, dort jedoch nicht mehr tätig. Zwei erwachsene Kinder.

- Klinischer Befund: Unauffällig, Body-Mass-Index (BMI) $26 \mathrm{~kg} / \mathrm{m}^{2}$; Blutdruck 110/70 mm Hg, Puls rhythmisch.

- Technische Untersuchungen: EKG, Echokardiographie, Spirometrie, Ergometrie unauffällig, vereinzelt ventrikuläre Extraystolen (VES).

- Diagnosen: Ausschluss einer strukturellen kardiopulmonalen Erkrankung; «funktionelle»Herzbeschwerden.

- Therapie: Wiederaufnahme und Pflege regelmässiger körperlicher auch sportlicher - Aktivitäten (durchlebte/durchseelte Bewegung).

- Medikation: Aconitum Napell D12 DI, Cimicifuga Comp. DIL N2, Aurum/Stibium/Hyoscyamus GLO N1 20 g, Cactus Comp. II GLO N1.

- Verlauf (Konsultation 23.06.2015): Es gehe wesentlich besser. Die klimakterischen Beschwerden sind kaum noch spürbar, der Druck in der Brust ist weg. Die Patientin kann kaum glauben, dass die paar Tröpfchen und Globuli dies bewirkt haben.

- Epikrise: Menschenkundlich ist bei den «funktionellen»Herzbeschwerden in der Terminologie Rudolf Steiners die geordnete Verbindung von Astralleib einerseits und Ätherleib/physischer Leib andererseits aufgehoben. Der Astralleib «verhakt» sich in der Herzregion, schafft «Bewusstsein am falschen Ort»; die Patientin fühlt sich bedroht. Der Wiederherstellung dieser geordneten Verbindung dienen die thera- peutischen Massnahmen; hier insbesondere viele gute Erfahrungen mit Aconit in mittlerer Potenz bei vom unteren Menschen aufsteigender Herzangst.

\section{Intermittierendes \\ Vorhofflimmern}

\section{R.M., 71 Jahre (w); Erstkonsultati-} on 22.09.2016

- Aktuelle Beschwerden: Im März 2016 Erstmanifestation einer absoluten Arrhythmie bei Vorhofflimmern, mit sehr hohen Frequenzen (bis 200/min). Unter Betablocker im Notarztwagen bereits spontane Kardioversion. Seither wiederholt Palpitationen; regelmässige körperliche Aktivitäten sind problemlos möglich.

- Frage der Patientin: Zweitmeinung bei intermittierendem Vorhofflimmern. Komplementärmedizinische Therapieoptionen? Antikoagulation?

- Weitere Vorgeschichte: Mit ca. 28 Jahren Appendektomie, mit Mitte 30 Verdacht auf Lungensarkoidose, mit 42 Jahren Hysterektomie und Ovarektomie.

- Kardiovaskuläres Risiko: Keins.

- Biografie: Geboren in Schlesien, die Mutter im Kindbett gestorben, grossgeworden bei einer polnischen Familie, Kindheit nicht in schöner Erinnerung (Arbeitskraft/ «Aschenputtel»). Heirat mit 21 Jahren, Geburt des ersten Kindes, Übersiedlung nach Deutschland. Erneute, zweite Ehe, aus dieser Ehe keine Kinder, Beziehung problematisch, mitgearbeitet im Betrieb des Ehemannes, seit 9 Jahren verwitwet, seither auch neue Beziehung, stabil. Mit dem plötzlichen Tod des Ehemanns schwere wirtschaftliche Probleme, äusserst belastend. Beschäftigt mit sportlichen Aktivitäten (Schwimmen/Fitness), kocht sehr gerne, Gartenarbeiten, Zeitunglesen.
- Klinischer Befund: Unauffällig, normoton, rhythmisch. BMI 22,5 $\mathrm{kg} / \mathrm{m}^{2}$.

- Technische Untersuchungen: EKG, Echokardiogramm, Gefässe: unauffällig. Kein Ischämiehinweis (Stressechokardiographie), allerdings $\mathrm{zu}$ Belastungsende Auftreten von Vorhofflimmern mit spontaner Kardioversion. Langzeit-EKG (7 Tage): keine Episode von Vorhofflimmern.

- Diagnosen: Intermittierendes Vorhofflimmern; Ausschluss einer strukturellen kardiovaskulären Erkrankung.

- Therapie: Fortsetzung und Pflege der regelmässigen körperlichen Aktivitäten, nach Möglichkeit Heileurhythmie.

- Medikation: Tambocor $100 \mathrm{mg}$, Isoptin $80 \mathrm{mg}$, Xarelto $20 \mathrm{mg}$ : «Pill in the Pocket» bei Bedarf. Argentum Met Praep D20 TRI (Msp), Oxalis Folium 30\% SAL (mittags Oberbauch einreiben, $30 \mathrm{~min}$ ruhen), Strophanthus Comp. GLO, Cardiodoron Mag Phos D3, Cichorium Pl Tot D1 DIL.

- Verlauf (Konsultation 13.12.2016): Es gehe sehr, sehr gut, wie seit Jahren nicht mehr. Wenig Palpitationen, sie sei ruhiger, ausgeglichener, die zurückliegenden Belastungen tangierten sie kaum noch.

- Epikrise: Grundsätzlich finden sich im Herzgeschehen «nur» Wirkungen: aus dem seelischen Bereich von aussen, vom Nerven-SinnesSystem und vom Stoffwechselbereich ausgehend; unter der Annahme, dass der wirtschaftliche Schock nach dem Tod des zweiten Ehemanns ursächlich mitverantwortlich sein kann, wurde in diesem Fall eine Therapie mit Oxalis und Silber eingeleitet (Silber hier: Wiederherstellung der Deformationen der höheren Wesensglieder mit der Folge einer vom Herzen ausgehenden beruhigenden Wirkung) [7]. Sehr oft gelingt beim Vorhofflimmern eine erhebliche symptomatische Besserung. 


\section{Koronare Herzkrankheit}

\section{P.H., 69 Jahre (m);}

Erstkonsultation 28.04.2015

- Aktuelle Beschwerden: Bei einer eher zufällig durchgeführten Ergometrie wurde vor 10 Tagen ein pathologischer Befund erhoben: komplexe ventrikuläre Rhythmusstörungen auf niedriger Belastungsstufe. Die unverzüglich durchgeführte Adenosin-Stress-Magnetresonanztomographie zeigte ein belastungsinduziertes Perfusionsdefizit anterolateral/inferolateral, Infarktnarbe inferior. Vonseiten des Herzens subjektiv beschwerdefrei, keine Angina-pectoris-Symptomatik; ausser Aspirin habe er die empfohlene übliche konventionelle Therapie nicht aufgegriffen, invasive $\mathrm{Di}$ agnostik lehne er ab.

- Frage des Patienten: Zweitmeinung; komplementärmedizinische Therapieoptionen?

- Weitere Vorgeschichte: Keine wesentlichen Vorerkrankungen.

- Kardiovaskuläres Risiko: Ein Onkel erlitt mit 70 Jahren einen Herzinfarkt, regelmässiger leichter $\mathrm{Zi}$ garillo-Konsum seit 30 Jahren.

- Systemanamnese: Gelegentlich kalte Füsse, seit einigen Monaten regelhaft Durchschlafstörungen, vermehrtes Traumleben, Präferenz für Salziges, Verdauung gut, Neigung zu Obstipation, weitsichtig.

- Biografie: Aufgewachsen im Grenzgebiet zu Belgien, ein älterer Bruder, schöne Kindheit. Gewerbeschule, Ausbildung zum Installateur, Hochschulreife: Ingenieurstudium in Versorgungstechnik. Seit 1975 selbstständig mit eigenem Ingenieurbüro, Wärmeversorgung von grossen Immobilienkomplexen, Handel mit Energie. Die Tätigkeit habe Freude gemacht, sei jedoch auch mit viel Ärger verbunden, ca. 12 h/Tag, 6-7 Tage/Woche. Verheiratet seit 44 Jahren, stabil, zwei Kinder, fünf Enkel.

- Klinischer Befund: Normoton, Puls $64 / \mathrm{min}$. VES; sonst unauffällig. BMI $23,5 \mathrm{~m} / \mathrm{kg}^{2}$.

- Technische Untersuchungen: Im EKG keine typischen Infarktresiduen, im Echo Bestätigung des bekannten Infarktareals, leichter Mitralklappenprolaps, im Stress-Echo bei $75 \mathrm{~W}$ zusätzliche Ischämien, komplexe ventrikuläre Rhythmusstörungen (Salven).

- Diagnosen: KHK mit abgelaufenem stummen Infarkt inferior, inferoseptal, belastungsinduzierte Ischämie, komplexe ventrikuläre Rhythmusstörungen.

- Therapie: Durchlebte/durchseelte Bewegung, künstlerische Tätigkeiten (Wiederaufnahme des Klarinettenspiels), komplettes Einstellen des Nikotinkonsums, drastische Reduktion des Arbeitspensums.

- Medikation: Stannum Met 0,4\% SAL (abends beruhigende Herzeinreibung), Belladonna Planta Tota D6 DIL im Wechsel mit Plumbum Mel. D10. Crataegus Comp. DIL
N2 $100 \mathrm{ml}$, ASS 100, Sarothamnus Comp AMP s.c., Aurum/Stibium/ Hyoscyamus AMP s.c. Arnica D12.

- Verlauf: Bei regelmässigen Kontrollen in drei- bis viermonatlichen Abständen bleiben die Infarktzeichen im Echo bestehen; im StressEcho sind zuletzt (24.11.2016) zusätzliche Ischämiezeichen oder komplexe ventrikuläre Rhythmusstörungen bei $100 \mathrm{~W}$ nicht mehr nachweisbar. - Die Verunsicherung ist fast komplett verschwunden.

- Epikrise: Therapieziel ist eine Verlangsamung des Skleroseprozesses, d.h. eine Reduktion der Dominanz des «oberen Menschen» mit Verfestigungs-/Sklerosetendenz, sowie die Stärkung der «Mitte», des rhythmischen Systems und der aufbauenden Kräfte. Diesem Ziel dienen die Allgemeinmassnahmen sowie das komplexe Medikamentenprogramm. Bei subjektiver Beschwerdefreiheit lehnt der Patient invasive Verfahren, auch ein konventionelles Medikamentenkonzept (ausser ASS 100) ab. Diese Position wird von mir mitgetragen, unter der Voraussetzung, dass die vorgeschlagenen therapeutischen Massnahmen konsequent implementiert werden. Dies gelingt dem Patienten, die Situation stabilisiert sich.

\section{Literatur}

1 Steiner R: Geisteswissenschaft und Medizin, ed 5. 5. Vortrag. GA 312. Dornach, Rudolf Steiner, 1976, pp 96-98.

2 Kaufmann C: Herzerkrankungen; in Girke M (Hrsg): Geriatrie. Grundlagen und therapeutische Konzepte der Anthroposophischen Medizin. Berlin, Salumed, 2014, pp 207-209.

3 Hötzel J: Krankengeschichten aus der kardiologischen Praxis; in Rubens C, Selg P (Hrsg): Das menschliche Herz: Kardiologie in der anthroposophischen Medizin. Dornach, Ita Wegmann Institut, 2014, pp 242-244.

4 Bopp A, Breitkreuz T: Bluthochdruck senken: Das 3-Typen-Konzept, ed 4. München, Gräfe \& Unzer, 2016.

5 Bopp A, Breitkreuz T, Fried A, Gruber J: Das Herz stärken - das ganzheitliche Programm, ed 3. München, Gräfe \& Unzer, 2013.
6 Kaufmann C: Das Herz im Mittelpunkt - anthroposophische Kardiologie. Heidenheim, Anthrosana, 2016, Heft Nummer 232.

7 Steiner R: Physiologisch-Therapeutisches auf Grundlage der Geisteswissenschaft, ed 3. 2. Vortrag. GA 314. Dornach, 1. Januar 1924. Dornach, Rudolf Steiner, 1989, p 197. 